\title{
Tsafon
}

Revue d'études juives du Nord

$72 \mid 2016$

Juifs, Israéliens, dans la littérature française et israélienne

\section{Entre condamnation de l'esprit juif et déférence à son égard, Wilhelm Emrich}

Helmut Pillau

\section{(2) OpenEdition}

Journals

Édition électronique

URL : https://journals.openedition.org/tsafon/379

DOI : $10.4000 /$ tsafon.379

ISSN : 2609-6420

Éditeur

Association Jean-Marie Delmaire

Édition imprimée

Date de publication : 1 décembre 2016

Pagination : 125-138

ISSN : $1149-6630$

Référence électronique

Helmut Pillau, «Entre condamnation de l'esprit juif et déférence à son égard, Wilhelm Emrich », Tsafon [En ligne], 72 | 2016, mis en ligne le 31 mai 2018, consulté le 27 juin 2021. URL : http:// journals.openedition.org/tsafon/379; DOI : https://doi.org/10.4000/tsafon.379 


\title{
Entre condamnation de l'esprit juif et déférence à son égard, Wilhelm Emrich
}

\author{
Helmut Pillau*
}

Note de la rédaction :

Notre collègue Helmut Pillau, de l'université Gutenberg de Mayence en Allemagne, a eu la gentillesse de nous communiquer un texte de Wilhelm Emrich (1909-1998), professeur de littérature allemande, qui milita au NSDAP sous le III ${ }^{\mathrm{e}}$ Reich. Nous le publions dans sa traduction française par Joseph Kolbl et il est suivi des remarques de Helmut Pillau qui fut son élève.

\section{Texte de Wilhelm Emrich (1943)}

\section{L'irruption de l'esprit juif dans la pensée scientifique et appliquée}

Jamais on ne pourra comprendre dans ses ultimes ramifications le danger mortel que l'irruption de l'esprit juif a également provoqué dans la littérature scientifique et appliquée, si l'on ne tente pas d'en déterminer avec plus de précision les présupposés et les modalités.

Il suffit de se représenter succinctement les grandes lignes de l'évolution intellectuelle au cours du $\mathrm{XIX}^{\mathrm{e}}$ siècle pour s'apercevoir avant tout de l'inexorable déclin des grands systèmes, des œuvres d'art et des

\footnotetext{
* Johannes Gutenberg Universität, Mayence, Allemagne. Traduction de l'allemand par Joseph Kolbl, université Charles de Gaulle - Lille 3.
} 
conceptions de la nature idéalistes que l'esprit allemand a engendré. Psychologisme, pensée causale mécaniste et matérialisme historique, tels sont les trois composants qui caractérisent cette décadence et dont les répercussions n'ont pas été tout à fait surmontées jusqu'à présent dans la littérature scientifique et spécialisée. Plus la lutte est âpre de nos jours pour parvenir à une nouvelle conception homogène empreinte de profondeur scientifique et d'une orientation idéologique claire, et plus sera déterminante la connaissance des armes dont s'est servi l'adversaire et dont il dispose toujours.

À revenir une nouvelle fois sur le point d'irruption par où s'est engouffré l'esprit judéo-marxiste dans l'univers mental allemand, on constate avec étonnement le choc violent entre une conception organiquement cohérente de la totalité et un intellectualisme juif qui dissout et nie en bloc toutes les valeurs spirituelles. Le système hégélien avait entrepris - fait unique dans l'histoire - d'interpréter tout l'univers de l'esprit, de la nature et de l'histoire et d'en percer les mystères. Or, au moment où celui-ci se décomposait graduellement sous l'effet des transformations opérées par les jeunes hégéliens, le juif Marx s'est engouffré dans les fissures et les brèches ainsi ouvertes pour transformer le système entier en utilisant habilement toutes les catégories créées par Hegel (méthodes dialectiques) en une vision matérialiste de l'histoire qui aujourd'hui encore constitue la bible du bolchevisme.

Ainsi l'ensemble des apports intellectuels, artistiques, scientifiques et techniques de l'humanité fut interprété, à la lumière d'une dialectique empruntée à Hegel, comme le simple reflet idéologique qui s'élève audessus d'une évolution purement économique. Cette conception se contente de considérer un courant important, tel que la Renaissance italienne, comme l'expression de l'économie précapitaliste qui produit, par le truchement d'une accumulation effrénée du capital, un état d'esprit libéré de toute entrave dogmatique traditionnelle. Dans ce contexte, les idéaux moraux de cette époque passent pour de simples masques habilement conçus, destinés à dissimuler le véritable état des choses. Il serait «dans l'intérêt» de la société concernée de créer de telles représentations pour abuser des masses qui désespèrent de la situation sociale. Cette conception aboutit fatalement à un scepticisme radical à l'égard de toute manifestation intellectuelle. Seule apparaît comme réelle la situation économique respective de la société humaine qui évolue en passant par des renversements dialectiques et des crises permanentes. On omet de poser la question de savoir comment s'explique l'évolution 
économique sans l'intervention de l'esprit humain; à moins de répondre en recourant à une mythologie économiste empreinte de fatalisme qui prétend que le processus économique se déroule de façon mystérieuse en vertu d'un automatisme dialectique, indépendamment de la volonté de l'individu et débouche en fin de compte sur une uniformisation totale de toutes les couches sociales et de tous les individus.

Il n'en va pas autrement de l'irruption de l'esprit juif dans la psychologie. Là aussi des juifs, tels que Siegmund [sic] Freud, Alfred Adler et d'autres ont réussi à s'infiltrer dans une position allemande affaiblie, résultat de la critique de la civilisation du $\mathrm{XIX}^{\mathrm{e}}$ siècle (Nietzsche, Kierkegaard), d'une psychologisation et d'une relativisation progressive des critères de la connaissance objective. Profitant de la dissolution de toutes les valeurs philosophiques fixes, les psychanalystes juifs ont fait accroire, pendant des décennies, aux larges masses de gens plus ou moins instruits que toutes les représentations, tous les actes intellectuels et moraux n'étaient que les manifestations et les illusions, voilant la réalité, d'une sphère pulsionnelle se déroulant à un niveau inférieur. Toute l'évolution culturelle de l'être humain, l'ensemble de sa production artistique, philosophique et religieuse furent interprétés comme des compensations d'une libido refoulée. Dans les deux cas, qu'il s'agisse du matérialisme historique (marxisme) ou de la psychanalyse, l'intention, la structure et l'objectif de l'esprit juif sont strictement identiques. On nie un monde de l'esprit et des valeurs morales en soi et le qualifie de masque d'une «réalité » sous-humaine. À l'instar de la philosophie de Spinoza qui nie complètement le libre arbitre et soumet la nature tout entière, voire l'Être suprême, à un déterminisme mathématique, la responsabilité personnelle de l'homme libre par sa volonté s'efface totalement dans les visions juives de la culture, les doctrines économiques, les théories psychologiques etc. juives actuelles. L'homme dans sa totalité est empêtré dans ses «intérêts » économiques et ses pulsions.

Il en découle des conséquences graves pour la littérature scientifique et appliquée. Ces théories marxistes et psychanalytiques, propagées dans une large mesure par des auteurs juifs, ont envahi les domaines de la médecine, la psychologie, la philosophie, les sciences de l'éducation, la science juridique, les lettres etc. Si l'on y associe les idées libérales de tolérance, de véritables crimes furent « expliqués» par leurs prétendues origines «économiques » ou «psychologiques » et partant excusés. Cette pensée juive ne connaît pas le concept de «culpabilité », 
chaque acte, chaque comportement humain étant déterminé par des forces collectives qui agissent en sous-main et dépassent la personne, que ce soit dans la vie sociale ou dans la sphère subconsciente des pulsions. Il s'ensuivit un laxisme inimaginable dans le système judiciaire.

Comme, par ailleurs, les juifs avaient la haute main sur tout le système de santé publique, par une habile politique de recrutement $(90 \%$ des hôpitaux et des établissements de santé publics étaient placés sous la responsabilité de médecins juifs), les conséquences pratiques pour l'hygiène publique furent désastreuses.

Si l'on est assurément parvenu à mettre un terme à ces abus, la lutte qui se joue au niveau intellectuel et scientifique est toujours âpre, son issue incertaine. Si jadis l'esprit judéo-marxiste a réussi, sur les débris de l'idéalisme allemand, à entamer sa marche triomphale à travers le monde, sa défaite définitive ne pourra intervenir que sur la base d'une nouvelle pensée totalisante qui soit à la hauteur de l'idéalisme allemand pour sa puissance intellectuelle, sa profondeur et sa dimension mondiale. Une telle pensée totalisante suppose une renaissance de l'esprit philosophique tout court. De même qu'à l'époque classique des découvertes techniques et scientifiques $\left(\mathrm{XVII}^{\mathrm{e}}\right.$ siècle) où les physiciens, les chimistes, les mathématiciens etc. se sont unanimement reconnus dans un seul philosophe (Leibniz, Descartes etc.), une interprétation homogène de notre réalité politique et intellectuelle ne sera pas concevable sans une imprégnation philosophique des différentes disciplines scientifiques. Car le dépassement de la critique juive de la culture et de l'esprit, qui s'est exprimée avec le plus de virulence dans le marxisme judéo-bolchevique et la psychologie juive, ne sera possible que par une interprétation systématique, englobant notre faculté de connaître dans son ensemble. « Le gouffre du relativisme » (voir E. May) nous menace toujours, et le psychologisme dans la gnoséologie n'est toujours pas surmonté. Un dépassement, qui résiste à l'examen scientifique, de ces courants n'est possible qu'en redéfinissant le concept de liberté à la fois sur la base des sciences exactes et sur celle des sciences humaines et de la philosophie. L'unité entre les sciences exactes et les sciences humaines, mise en pièces au début du $\mathrm{XX}^{\mathrm{e}}$ siècle par des distinctions philosophiques dues par exemple au juif Husserl, ne peut être rétablie qu'en mettant en contact, pour prendre un exemple, les résultats surprenants de la physique nucléaire moderne avec les problèmes très pointus de l'ontologie philosophique moderne et de la philosophie de l'histoire. Pour éloignées que soient ces disciplines, elles ne se recoupent pas moins dès lors qu'on 
s'interroge sur la relation entre liberté et causalité. Les sciences humaines, quant à elles, ont réussi, dans le débat avec l'historicisme du $\mathrm{XIX}^{\mathrm{e}}$ siècle, à préserver au phénomène historique, malgré tous les liens de causalité, sa spécificité qui permet de comprendre, d'un point de vue ontologique, sa liberté, sa valeur absolue et intemporelle; de la même façon, l'une des tâches primordiales de la science consistera à déterminer de façon homogène le véritable rapport entre manifestations intellectuelles et économiques, entre le conscient et l'inconscient. Ce n'est que de cette manière que l'intrusion pernicieuse des juifs dans les disciplines les plus diverses pourra être enrayée et éliminée et que l'idéal germanique de liberté pourra avoir définitivement raison du déterminisme mécaniste.

\section{$* * * * * * * * * * * * *$ \\ Entre condamnation de l'esprit juif et déférence à son égard, Wilhelm Emrich}

\section{par Helmut Pillau}

Ayant été en contact, pendant longtemps, avec Wilhelm Emrich depuis ma scolarité à Berlin -, les révélations tardives sur ses activités sous le $\mathrm{III}^{\mathrm{e}}$ Reich et notamment ses dérapages antisémites m'ont profondément choqué. Il serait ainsi inconvenant, me semble-t-il, de passer sous silence la dimension personnelle en abordant cette question. J'aimerais donc, en commentant les faiblesses d'Emrich, les intégrer dans un récit de mes propres expériences.

C'est mon professeur d'allemand qui a attiré mon attention sur lui au cours de la dernière année que j'ai passée au lycée. C'est elle qui m'a invité à l'accompagner pour assister à une manifestation sur Heinrich von Kleist dans le bâtiment de "l'Académie protestante" sur les bords du Kleiner Wannsee, tout près de l'endroit où, en 1811 , le poète s'est donné la mort avec sa compagne d'infortune. Au centre de cette manifestation était placée une conférence de Wilhelm Emrich qu'il publiera plus tard sous le titre «Heinrich von Kleist: estime de soi, une obligation ».

\footnotetext{
${ }^{1}$ Cette manifestation, organisée par la « Heinrich-von-Kleist-Gesellschaft», eut lieu, en 1961, dans le cadre de la commémoration du $150^{\mathrm{e}}$ anniversaire de la mort de Kleist (1777- 1811).
} 
Même s'il est certain qu'à cette époque je ne comprenais pas tout, c'est la prestance de l'orateur qui m'a laissé une impression durable. En essayant ici de retrouver les raisons de cette fascination, je tenterai de mettre en lumière les caractéristiques de cet homme.

À ce moment il me faisait l'effet de quelqu'un qui, après avoir atteint un sommet au bout d'un effort considérable, cherche à défendre cette hauteur coûte que coûte. On retrouvait cette attitude dans sa diction, catégorique, emphatique, sa façon de marteler les mots par moments. Dès lors qu'il parlait de "réalité empirique », on percevait très nettement une note péjorative. C'est précisément d'elle qu'il fallait s'affranchir pour parvenir à atteindre cette altitude convoitée. Certes, il ne donnait pas l'impression d'être communicatif en maltraitant ceux qui étaient restés en contre-bas. Au lieu de chercher un échange avec ses semblables, il préférait les mettre face à sa propre expérience des sommets. Au premier abord, cette attitude avait quelque chose de déconcertant, mais on finissait par accepter ce sérieux, cette exaltation qui sont le propre d'un prêtre. Peut-être pouvait-on parler, en l'écoutant, d'un culte voué à la connaissance supérieure ou à l'esprit. Tel un prêtre, il effrayait son auditoire par un diagnostic inquiétant des temps présents. Il semblait proférer des visions sombres quand, au cours de son exposé (je viens de le vérifier dans le texte), il déplorait en bloc « disparition progressive de l'esprit et de l'âme, ainsi que la réification au sein du monde empirique $»^{2}$. Tout ceci à seule fin de souligner l'extraordinaire mérite du poète. Cette antithèse entre dénonciation et élévation se reflète d'ailleurs dans le titre d'un de ses livres, aux accents religieux : «Protestation et prophétie ».

L'incapacité de Kleist de trouver un arrangement avec la langue usuelle, avec la vie pratique, voire la vie tout court, est glorifiée par Emrich qui y voit l'expression d'une inflexibilité intellectuelle sans concession. Kleist ne voulait pas se résigner à accepter que la vie se limite à un état de somnolence. Même si l'estime de soi et la responsabilité personnelle n'étaient accessibles qu'au détriment du bienêtre, il préférait ces exigences au bien-être. Qui plus est, Emrich réaffirmait cette attitude en faisant un rapprochement entre Kleist et un autre écrivain célèbre : Kafka, sans conteste un admirateur de l'œuvre du

\footnotetext{
${ }^{2}$ Wilhelm Emrich, «Heinrich von Kleist: Selbstbewusstsein als Pflicht» [Estime de soi, une obligation] dans Wilhelm Emrich, Geist und Widergeist. Wahrheit und Lüge in der Literatur. Studien [L'esprit et sa négation. Vérité et mensonge en littérature. Études]. Frankfurt / Main, Athenäum, 1965, p. 130.
} 
premier. Kleist, selon lui, était aussi «moderne » que Kafka dans la mesure où, dans les deux cas, un fossé infranchissable séparait leur message des moyens d'expression dont disposait chacun d'eux. Leur langue était extrêmement dépouillée sans qu'on puisse encore accorder un crédit à cette objectivité. Et il y avait bien une intention appellative dans cette façon de qualifier ces deux grandes figures de la littérature de pionniers d'une spiritualité absente, mais non moins indispensable. Le jeune lycéen que j'étais alors, pris de court, en fut ébloui. On pouvait se sentir honteux ou, au contraire, secoué lorsque Emrich, à la fin de sa conférence, parlait d'une «élite intellectuelle», d'une «aristocratie intellectuelle » ou, en reprenant un mot de Kafka, de « noblesse $\rangle^{3}$. Mais cette élite, dans ces conditions, restait nécessairement isolée, d'une manière presque fatale.

Emrich m'impressionnait, et j'étais loin d'être le seul. Dans un article nécrologique, le germaniste Hartwig Schultz allait le qualifier - à raison, me semble-t-il - d'« enthousiasmeur ${ }^{4}$. Il était ainsi naturel de me rapprocher de lui dans le cadre de mes études et de suivre en priorité ses cours d'une portée immense. Un heureux hasard voulut que cet éminent spécialiste de Gœthe qui avait publié un ouvrage sur «Le symbolisme dans Faust II » proposait, dès mon arrivée à l'université, un cours sur Gœthe. Les auditeurs, non seulement des étudiants et des germanistes, se rendaient en masse dans le grand amphithéâtre pour l'écouter. Par ailleurs, il s'adressait à un public plus large dans des conférences « du soir » et publiait de temps à autre des contributions dans un quotidien. Certains toutefois parlaient en catimini du regard démoniaque de cet homme de grande taille, aux cheveux noirs et au pied bot; d'autres le qualifiaient de «preneurs d'hommes » [Menschenfänger]. Ses séminaires étant sans cesse pléthoriques, je me suis contenté de suivre un « séminaire de recherche » sous sa direction qui s'intitulait « Théories du langage, de Herder à Grimm ». Mon exposé était consacré au philosophe chrétien Johann Georg Hamann.

Mais lorsqu'à la rentrée 1965 une autre sommité de la théorie de la littérature, le juif d'origine hongroise Peter Szondi, fit son apparition à

\footnotetext{
${ }^{3}$ Ibid. p. $145 \mathrm{sq}$.

${ }^{4}$ Hartwig Schultz, Begeisterer. Wilhelm Emrich ist tot [Enthousiasmeur. À l'occasion du décès de W. Emrich], dans la Frankfurter Rundschau du 13 août 1998. Cet article nécrologique se termine de la façon suivante: "Ainsi il restait à Berlin le seul germaniste qui, même après le mouvement des étudiants, jouissait d'une grande renommée et qui était capable de fasciner jusqu'à une date récente. Même en tant que professeur émérite il remplissait encore le grand amphithéâtre ».
} 
l'Université libre de Berlin, je quittai les Études germaniques pour m'inscrire dans une discipline nouvellement créée, la Littérature générale et comparative. J'étais motivé par l'idée de me détacher de la littérature allemande et de l'idéalisme allemand - principale source d'inspiration d'Emrich - et de m'ouvrir à des horizons plus larges. En même temps, Szondi me semblait prolonger, mais avec une démarche méthodologique plus réfléchie, plus subtile, ce que j'avais appris chez Emrich. Alors que ce dernier, grâce à son diagnostic des temps présents, empreint de désespoir, semblait appartenir à la mouvance d'Adorno et de Benjamin, Szondi faisait partie du cercle intime autour d'Adorno, de l'École de Francfort donc. Ces années étaient très mouvementées, en raison des événements de 1968 notamment. Le suicide de Szondi, en 1971, mit brutalement fin à cette période et, harcelé de remords, je retournais après toutes sortes de détours chez Emrich, là où j'avais commencé mes études. C'est sous sa direction que j'allais terminer ma thèse de doctorat sur le rapport entre la conception dramaturgique de Schiller et la théorie de la tragédie de Hegel. Nos relations étaient tout à fait simples et cordiales.

Ainsi, une page semblait se tourner, d'autant que je commençai à enseigner la littérature générale et comparative à l'université de Mayence en 1980. Mais lorsque Emrich mourut en 1998, à l'âge de 88 ans, je me trouvai inopinément une nouvelle fois confronté à lui. On parlait alors beaucoup d'un roman à clefs de Kurt Mautz, un ami de jeunesse d'Emrich: Der Urfreund ${ }^{5}$, dans lequel il aborde les revirements opportunistes d'Emrich sous le III $^{\mathrm{e}}$ Reich. Certaines citations tirées d'un pamphlet antisémite m'effrayèrent. J'étais très mal à l'aise parce que ces révélations ne cadraient pas avec l'idée que je me faisais de lui. Emrich qui s'inspirait d'Adorno et de Benjamin, qui avait fait une thèse dirigée par le juif allemand Martin Sommerfeld et qui, plus tard, vénérait tant Kafka, cet homme serait antisémite ? Sans les impulsions reçues lors d'un séminaire dirigé par Adorno en 1932, toute son œuvre scientifique serait inimaginable - c'est du moins ce qu'estimait Szondi qui l'a qualifié «d'élève d'Adorno et de Benjamin $»^{6}$. Dans un premier temps, je ne cherchai pas à en savoir davantage puisque plusieurs personnes assez

\footnotetext{
${ }^{5}$ Der Urfreund, [L'Ami originel]. Roman. Paderborn, Igel-Verlag, 1996.

6 Peter Szondi, Einführung zu Adornos Vortrag «Zum Klassizismus von Goethes Iphigenie » am 7. 7. 1967 [Introduction à la conférence d'Adorno du 7 juillet 1967 « À propos du classicisme de l'Iphigénie de Goethe »] dans Peter Szondi, Über eine "Freie (d.h. freie) Universität ». Stellungnahmen eines Philologen [Au sujet d'une « Université Libre (c.-à-d. vraiment libre) ». Prises de position d'un philologue]. Frankfurt / Main, Suhrkamp, 1973, p. 56.
} 
médiocres de mon entourage s'offusquaient de l'affaire pour des raisons tout à fait intéressées. Je ne devais lire ce roman qu'en 2013 et c'est à ce moment-là que je commençai à m'occuper de cette histoire embarrassante. Au début : plusieurs longues conversations téléphoniques avec l'acteur Rolf Mautz, le fils de Kurt Mautz, où il était question des relations de son père avec Emrich. Il s'ensuivit une réflexion sur cette thématique en quatre étapes.

Dans un premier temps, je cherchai à comprendre la position ambiguë d'Emrich en la plaçant dans le cadre général des rapports ambivalents entre christianisme et judaïsme. On sait que le christianisme, au lieu de se situer d'emblée dans une opposition au judaïsme, naissait de controverses à l'intérieur du peuple juif. Mais à partir du moment où la nouvelle religion cherchait à s'affirmer par rapport à la religion juive, elle risquait de se couper de ses racines, danger que l'apôtre Paul avait déjà souligné de façon prémonitoire ${ }^{7}$. Pour se régénérer, il lui était nécessaire de retourner aux sources ; c'est ce qu'illustre particulièrement la Réforme luthérienne. Tenu en suspicion par l'Église traditionnelle, Luther eut recours à la Bible et aussi à la langue hébraïque qu'il affectionnait. Cette démarche n'entraîna pas pour autant chez lui une plus grande gratitude à l'égard du judaïsme, mais à l'opposé une volonté accrue, même hystérique, de s'en démarquer. Cette proximité retrouvée avec ses propres racines n'est pas ressentie uniquement comme bénéfique, mais aussi comme une menace. Le risque était réel de voir apparaître l'indépendance, postulée, du christianisme par rapport au judaïsme comme imaginaire, du moins tendanciellement. Ainsi le christianisme n'a jamais pu surmonter les hésitations entre deux positions : se rapprocher du judaïsme, attitude que la spiritualité réclamait, ou s'en démarquer de façon polémique. Trahir ses origines spirituelles était pour le christianisme à la fois un acte de libération et une hypothèque. C'est précisément, me semble-t-il, cette ambivalence qui se reflétait, mais sous les auspices de la sécularisation, dans l'attitude sans cesse hésitante d'Emrich entre appréciation du judaïsme et son rejet.

\footnotetext{
${ }^{7}$ «Si tu te glorifies, sache que ce n'est pas toi qui portes la racine, mais que c'est la racine qui te porte $»$. (Romains 11, 18. Traduction L. Segond)
} 
C'était pour moi une expérience enrichissante de ne pas m'en tenir à ces spéculations en voulant élucider le cas d'Emrich. La participation à un atelier consacré à Wilhelm Emrich, qui s'est tenu en février 2016 à l'université Humboldt de Berlin sous la direction du professeur Jörg Schönert, m'a fait avancer. Des historiens principalement et quelques théoriciens de la littérature y faisaient part de leurs recherches sur les revirements d'Emrich, passant de la gauche radicale au nazisme, ses activités professionnelles sous le III $^{\mathrm{e}}$ Reich et notamment sa fonction particulièrement compromettante de censeur au ministère de l'Information du peuple et de la Propagande, sous les ordres de Joseph Goebbels donc. C'est dans le cadre de cette activité que son pamphlet antisémite allait voir le jour. Mais auparavant, il avait été chargé par ce même ministère d'établir une bibliographie, à la Librairie allemande de Leipzig ["Deutsche Bücherei Leipzig »], des publications d'auteurs juifs en langue allemande. Les recherches historiques présentées dans cet atelier étaient intéressantes aussi par le fait qu'elles ne se limitaient pas à la personne de Wilhelm Emrich. On se penchait également sur d'autres chercheurs, tels que l'historien Theodor Schieder, le philosophe Erich Rothacker et l'historien de la littérature est-allemand Erich Kühne. Tous ont réussi après la guerre, malgré leurs activités compromettantes durant le III $^{\mathrm{e}}$ Reich, à faire une carrière remarquable dans une université allemande.

J'appris ainsi que Emrich fut interné par les Américains immédiatement après la fin de la guerre. Il s'en sortit sans trop de mal de la procédure devant une «chambre d'épuration». Grâce à des interventions, comme celle du germaniste allemand d'origine juive Oskar Seidlin, installé aux États-Unis, qui, dans un geste magnanime, intercéda en sa faveur, il fut classé dans la catégorie des personnes « déchargées de leurs fautes » [entlastet] et put aussitôt se lancer dans une brillante carrière universitaire. Au cours de son procès, il tenta d'ailleurs de se donner pour un adversaire du régime national-socialiste. Il ne fait pas de doute qu'il profitait, comme tant d'autres qui se trouvaient dans la même situation que lui, du déclenchement, consécutif au blocus de Berlin en 1948, de la guerre froide entre l'Union soviétique et les puissances occidentales. 
Situer le cas d'Emrich dans le contexte de son époque m'a aidé à mieux cerner un trait de son caractère, à savoir cette exaltation qu'il affichait dans l'arène universitaire. À présent, je voyais plus clairement que cet élan de rigorisme moral pouvait aussi servir à masquer ses écarts de conduite : comme toute la lumière était faite au niveau supérieur des grands principes, on pouvait bien se dispenser de s'attacher aux menus détails de la « réalité empirique ». Et l'adaptation d'Emrich à l'idéologie antisémite des nazis, justifiée d'ailleurs par des raisons uniquement tactiques, pouvaient en faire partie ${ }^{8}$. Dans un texte succinct, «Franz Kafka, un portrait », je tombai sur le passage suivant :

Le respect des principes moraux que chacun de nous s'impose, rendrait la vie impossible. Les idéaux, selon l'opinion commune, sont irréalisables. On les sacrifie avec « soulagement» pour pouvoir «s'en sortir». Le monde absurde de Kafka reflète la logique de notre vie. ${ }^{9}$

Aurait-il pensé aussi à lui-même en écrivant cela, à ses manquements sous le $\mathrm{III}^{\mathrm{e}}$ Reich ? Il établirait alors un parallèle entre son attitude et la fatale «logique de notre vie ». Comme tous les autres, il aurait été forcé de sacrifier « ses propres principes moraux » pour assurer sa survie. Il est évident toutefois que, ce faisant, il n'appartenait pas à l'élite qu'il ne cessait d'invoquer.

Dans sa conférence de 1961 sur Kleist, Emrich avait loué Kafka pour avoir dévoilé, dans le même esprit que Kleist, que l'attitude virtuose, mais sans cesse hésitante, des intellectuels modernes face à la relativité des convictions idéologiques n'était qu'un «sursis, une protection contre l'absolu » ${ }^{10}$. Or, à l'inverse, cette perspective de « l'absolu » devait servir à Emrich de protection contre les contingences embarrassantes, comme par exemple ses faux pas sous le III ${ }^{\mathrm{e}}$ Reich.

\footnotetext{
${ }^{8}$ Le fils d'Emrich, Hinderk Emrich, en tout cas a tenté d'interpréter l'attitude de son père pendant le $\mathrm{III}^{\mathrm{e}}$ Reich comme une adaptation purement tactique en vue d'augmenter sa marge de manœuvre pour des activités subversives. Cf. son courrier des lecteurs publié dans la Frankfurter Allgemeine Zeitung du 10 septembre 1998, p. 12.

${ }^{9}$ Wilhelm Emrich, «Franz Kafka : Porträt», dans Geist und Widergeist, p. 293, voir note 2 .

${ }^{10}$ Wilhelm Emrich, « Selbstbewusstsein als Pflicht » dans Geist und Widergeist, p. 146, voir note 2 .
} 
Il serait cependant trop facile, à mon sens, de prendre cette rhétorique emphatique pour un simple trait de caractère individuel. Car, ce faisant, il s'était mis au diapason de ce qui était attendu en haut lieu vers la fin des années cinquante et au cours des années soixante en Allemagne de l'Ouest. Emrich se conformait ainsi à la façon purement abstraite dont on avait coutume alors «d'assumer le passé ». Elle restait abstraite dans la mesure où elle se limitait au niveau de la moralité et de la philosophie de l'histoire. On n'acceptait guère l'idée de s'interroger sur la responsabilité individuelle de chacun pour les actes commis durant le III $^{\mathrm{e}}$ Reich. C'est pourquoi le besoin d'une catharsis devait se réduire à un rituel pathétique en guise de catharsis. Reconnaître sa faute, mais uniquement à ce niveau abstrait et théorique, n'était qu'une autre façon de la passer sous silence ${ }^{11}$.

Mais ce rituel d'une purification intérieure que l'État encourageait d'ailleurs, devait s'effondrer dès lors qu'il se trouvait confronté aux actes commis par des personnes qui avaient joué un rôle sous le $\mathrm{III}^{\mathrm{e}}$ Reich ; par exemple parce que la génération suivante, celle de 1968, posait des questions embarrassantes. Alors les propos incantatoires se révélaient soudain n'être que des subterfuges pour cacher sa propre conduite jusquelà passée sous silence. Cette pose grandiloquente de maître spirituel n'avait apparemment d'autre fonction que de dissimuler un passé pitoyable. On imagine ainsi l'immense choc qu'a dû éprouver, peu de temps avant sa mort, Wilhelm Emrich suite aux révélations contenues dans le livre de fiction de Kurt Mautz: Der Urfreund. On y trouve des citations tirées du pamphlet antisémite d'Emrich.

À ce propos, je pense à une courte lettre que Wilhelm Emrich avait jointe, le 25 décembre 1974, à l'envoi de son rapport sur la première partie de ma thèse. À la fin, il aborde ma manière de traiter le «mal» dans l'Esthétique de Hegel. Il se dit intéressé parce que le mal n'était pas seulement, comme je l'avais présenté, un problème de «l'individualité sans fond » mais aussi, l'exemple du fascisme le démontrait, un problème de l'Histoire.

Cette remarque indique la façon dont il entend faire face au fiasco du III $^{\mathrm{e}}$ Reich, et implicitement à la Shoah en particulier - et il ne songe pas un instant que Hegel ne se prête guère à ce genre d'exercice. En cherchant à interpréter les noirs desseins des nazis comme l'irruption du mal dans l'histoire, les plaçant de ce fait au niveau abstrait de la

\footnotetext{
${ }^{11}$ Jörn Rüsen parle de «Beschweigen» [Note du Traducteur]: jeu de mot avec «bereden » = aborder un sujet].
} 
philosophie de l'histoire, il parvient à les tenir à distance. La réflexion générale, par son degré d'abstraction aboutirait dans ce cas (comme potentiellement dans sa façon de traiter les œuvres littéraires) à une généralisation aux traits nébuleux plutôt qu'à des distinctions claires et détaillées. Si Emrich s'est qualifié à plusieurs reprises de « Grand Pan », il semble afficher, non sans une dose d'autodérision, une certaine préférence pour une conception visionnaire au détriment de la méticulosité. L'impression qu'il produisait sur son public démontre toutefois qu'il ne s'agit pas uniquement d'un trait de caractère individuel.

Ce n'est qu'au début de l'année 2016, dans le cadre de l'atelier mentionné, que je devais prendre connaissance du texte intégral du pamphlet d'Emrich: «L'irruption de l'esprit juif dans la pensée scientifique et appliquée ${ }^{12}$. À lui seul, ce titre fait l'effet d'une infamie. Il pourrait laisser à penser que les juifs sont assimilés à des cambrioleurs qui pénètrent dans la demeure bien entretenue de la science allemande.

Ce qui m'a répugné en particulier, c'était sa façon de reprendre à son compte sans vergogne les diffamations des juifs par les nazis, coutumiers du fait - et ce en 1943, au moment donc où le génocide des juifs battait son plein. Ainsi est-il question du « choc violent entre une conception organiquement cohérente de la totalité et un intellectualisme juif qui dissout et nie en bloc toutes les valeurs spirituelles $\gg{ }^{13}$. Alors qu'il fait passer les juifs pour les véritables instigateurs de la pensée moderne qui se diversifie sans entrave - et dans ce sens elle est en effet destructrice -, il fait l'éloge de l'idéalisme allemand pour sa «pensée totalisante ». Il s'indigne de voir Sigmund Freud et Karl Marx, en réduisant les hommes à de simples fonctions de processus anonymes, ne plus les considérer comme maîtres d'eux-mêmes. Or, le défi pour la culture allemande consisterait à libérer les hommes, dans l'esprit de l'idéalisme allemand, des griffes de ces puissances anonymes. Et il va jusqu'à invoquer une « liberté germanique ». Dans ce contexte, il ne s'agit pas pour lui d'un simple retour à l'idéalisme allemand; il songe plutôt à

12 Wilhelm Emrich: «Der Einbruch des Judentums in das wissenschaftliche und fachliche Denken » dans Das deutsche Fachschrifttum, 1943, Heft 4/5/6, pp. 1-2.

${ }^{13}$ Ibid. p. 1. 
une synthèse des acquis scientifiques de l'époque moderne (comme par exemple la physique nucléaire) et de la «pensée totalisante » allemande.

Un coup d'œil sur ses travaux ultérieurs montre qu'il est resté fidèle à cette vision, en tout cas à ses idées fondamentales. Mais en l'occurrence, c'est précisément un juif, Franz Kafka, qui devait répondre à ces exigences rigoureuses, du moins dans le domaine de la littérature. Kafka, selon Emrich, souscrivait sans réserve au monde moderne et à la relativisation des valeurs, mais ne capitulait pas devant lui, comme la plupart des autres. Il n'a donc pas renoncé à « l'absolu ». Au lieu de le rendre accessible en transcendant la réalité par le biais de la religion, il y parvient en s'imprégnant de la réalité, en particulier celle de l'homme. C'est en son sein qu'il découvrit l'absolu qui reste toutefois « informulable ${ }^{14}$. Le livre d'Emrich sur Kafka montre qu'il attribue cet exploit à la judéité de l'écrivain. Dans une note, il renvoie à :

... la forte attirance de Kafka pour le hassidisme des juifs de l'Est, depuis environ 1911-1912 et, d'une manière générale, pour la mystique juive dans laquelle, comme on sait, un rigorisme éthique était indissolublement lié à la conviction que le divin ou plutôt l'exigence absolue de Dieu résidait dans l'homme luimême. $^{15}$

Il semble bien qu'Emrich cherchait à effacer, par cette appréciation de l'esprit juif, son jugement antérieur, diamétralement opposé. Mais, faute de s'en être expliqué ouvertement, il portera à jamais les marques de son méfait.

\footnotetext{
${ }^{14}$ Wilhelm Emrich, Franz Kafka, Frankfurt / Main, Bonn, Athenäum-Verlag, 1965, $4^{\mathrm{e}}$ édition, p. 56.

${ }^{15}$ Ibid. p. 423. Emrich se réfère dans cette note - sans doute un ajout postérieur - au livre de Gershom Scholem, Die jüdische Mystik in ihren Hauptströmungen [La mystique juive à travers ses principaux courants]. Frankfurt / Main, Alfred Metzler Verlag, 1957.
} 